\title{
QUANTITATIVE CHARACTERIZATION OF MICROSTRUCTURE OF PURE COPPER PROCESSED BY ECAP
}

\author{
Ondřej ŠEdivÝ凶,1, Viktor Beneš¹, Petr PonížIL ${ }^{2}$, Petr KRÁL ${ }^{3}$ AND VÁClaV \\ SKLENIČKA ${ }^{3}$ \\ ${ }^{1}$ Department of Probability and Mathematical Statistics, Faculty of Mathematics and Physics, Charles \\ University in Prague, CZ-18675 Prague, Czech Republic; ${ }^{2}$ Department of Physics and Material Engineering, \\ Faculty of Technology, Tomas Bata University in Zlín, CZ-76272 Zlín, Czech Republic; ${ }^{3}$ Institute of Physics of \\ Materials, Academy of Sciences of the Czech Republic, CZ-61662 Brno, Czech Republic \\ e-mail: sedivy@karlin.mff.cuni.cz, benesv@karlin.mff.cuni.cz, ponizil@ft.utb.cz,pkral@ipm.cz, \\ sklen@ipm.cz
}

(Received August 9, 2012; revised February 4, 2013; accepted March 17, 2013)

\begin{abstract}
Orientation imaging microscopy (OIM) allows to measure crystallic orientations at the surface of a material. Digitalized data representing the orientations are processed to recognize the grain structure and they are visualized in crystal orientation maps. Analysis of the data firstly consists in recognition of grain boundaries followed by identification of grains themselves. Knowing the grain morphology, it is possible to characterize the grain size homogeneity and estimate structural parameters related to the physical properties of the material. The paper describes methods of imaging and quantitative characterization of the grain boundary structure in metals based on data from electron backscatter diffraction (EBSD). These methods are applied to samples of copper processed by equal-channel angular pressing (ECAP).
\end{abstract}

Keywords: electron backscatter diffraction, equal-channel angular pressing, random marked sets, second-order analysis.

\section{INTRODUCTION}

In microstructural research of metallic materials, methods allowing direct evaluation of the grain structure are often limited to investigation of $2 \mathrm{D}$ sections. Planar quantities are used to estimate parameters of the original 3D structure with usual stereological methods. One general limitation is that the estimators of the structural parameters are highly influenced by the resolution given by the grid step of the subsample where the measurements are provided. On the other hand, given the experimental setting, one has comprehensive information about the orientations in grains and disorientations in grain boundaries which can be further analysed with various methods. This paper presents basic principles of processing data from 2D scanning electron microscopy (SEM), describes methods of quantitative characterization of the observed microstructure based on second-order analysis of random marked sets and demonstrates the methods on particular samples.

To describe the structure of polycrystalline materials, modern attitude consists in the characterization of different types of boundaries present in the material and their connectivity in the grain boundary network (Rohrer, 2011). With data from 3-dimensional electron backscatter diffraction
(EBSD; Calcagnotto et al., 2010; Wilkinson and Britton, 2012) it is possible to explore the entire 5parametrical distribution of the grain boundaries where three parameters are related to the disorientation and the other two represent orientation of the interface plane. Despite of limitations of 2-dimensional observations, it still provides great potential for statistical analysis and it allows to process greater amount of observations than space- and timeconsuming 3D methods. It is also possible to estimate the 3-dimensional microstructure on the basis of statistical analyses of the data obtained from the 2dimensional EBSD.

Electron backscatter diffraction is a scanning electron microscope (SEM) based technique which has become well known as a powerful and versatile experimental tool for materials scientists, physicists, geologists and other scientists and engineers (Randle, 2009). It allows the measurement of microtexture (Jiang et al., 2008), microstructure quantification (Bastos et al., 2006), grain and phase boundary characterization (Randle et al., 2008), phase identification (Perez et al., 2006) and strain determination (Britton and Wilkinson, 2012) in crystalline multiphase materials of any crystal structure. 
The aim of the present paper is a systematic characterization of $\mathrm{Cu}$ processed by equal-channel angular pressing (ECAP) before creep testing. The creep behaviour of ECAPed materials probably belongs to the fewest examined properties of materials processed by ECAP. Creep behaviour was usually investigated in materials which were prepared by severe plastic deformation (SPD) technique at room temperature. For this reason, the creep tests were performed at higher temperatures than the processing temperature of SPD technique was. It is generally accepted that tensile samples are put into the furnace interior and then heated to the creep temperature. It is important to note that each specimen was heated to the testing temperature in the furnace of the creep testing machine up to creep temperature. For this reason, the microstructure is statically annealed and significantly influenced by temperature-induced changes like grain growth, recovery, recrystallization etc.

The systematic characterization of microstructure in $\mathrm{Cu}$ specimens processed by ECAP before loading can be important for better understanding of the unusual creep behaviour of ECAPed $\mathrm{Cu}$ (Dvořák et al., 2010). Despite of extensive interest in SPD material, there is no systematic work describing real microstructure of ECAP materials before loading when testing temperature in the creep testing machine was reached and stabilized.

To describe the microstructure, statistical methods characterizing spatial distribution of the boundaries marked by their disorientations are introduced. They basically work with a single mark determined by the disorientation angle $\theta$ but even more complex information about the disorientation can be used by distinguishing different types of "special" boundaries. The methods are applied on samples of copper processed by ECAP followed by annealing with different times and temperatures. Effect of different number of passes on the grain structure was examined in previous studies (Ilucová et al., 2007; Král et al., 2011).

The rest of the paper is organized as follows. The next section describes the experimental setup including preparation of the samples and their microscopical observations. The following section introduces basics of image processing of these observations. Further, the processed data are used for quantitative analysis of grain boundaries and this analysis is followed by numerical results. Conclusions are made in the final section.

\section{EXPERIMENTAL BACKGROUND}

The microstructure of specimens was examined by scanning electron microscope Jeol 6460 equipped with an EBSD unit operating at an accelerating voltage of $20 \mathrm{kV}$ with specimen tilted at $70^{\circ}$. Results presented in the paper come from the research of copper (99.99\% purity) processed by ECAP which involves pressing of a sample through a die within a channel that is bent into an L-shaped configuration (Fig. 1).

The billets in the cast state with $10 \mathrm{~mm} \times 10 \mathrm{~mm}$ cross section and $60 \mathrm{~mm}$ length were processed by ECAP at room temperature using a die with two perpendicular channels. Each billet was processed by a selected number of ECAP passes. The ECAP was performed using route $\mathrm{Bc}$ (Furukawa et al., 1998) in which the billet was rotated around the longitudinal axis by $90^{\circ}$ clockwise between the passes. Each pass corresponds to an additional strain value approximately equal to 1 . After ECAP, billets were annealed at $373 \mathrm{~K}, 423 \mathrm{~K}, 473 \mathrm{~K}$ or $573 \mathrm{~K}$ for 10 hours. The microstructure analyses were focused on samples processed by 8 ECAP passes. The microstructure changes of pure $\mathrm{Cu}(99.99 \%)$ processed by 8 ECAP passes occurring during the annealing caused considerable decrease of hardness (Fig. 2).

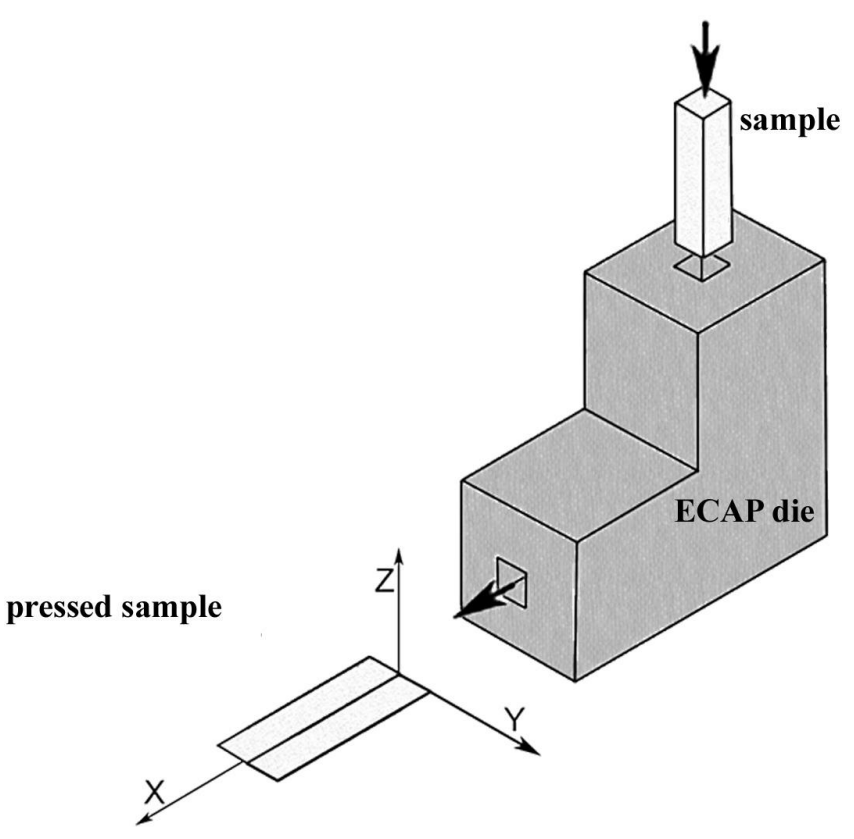

Fig. 1. Scheme of equal-channel angular pressing (ECAP) facility with definitions of the directions $X, Y$ and $Z$. A sample of metallic material is pressed through an L-shaped channel. 


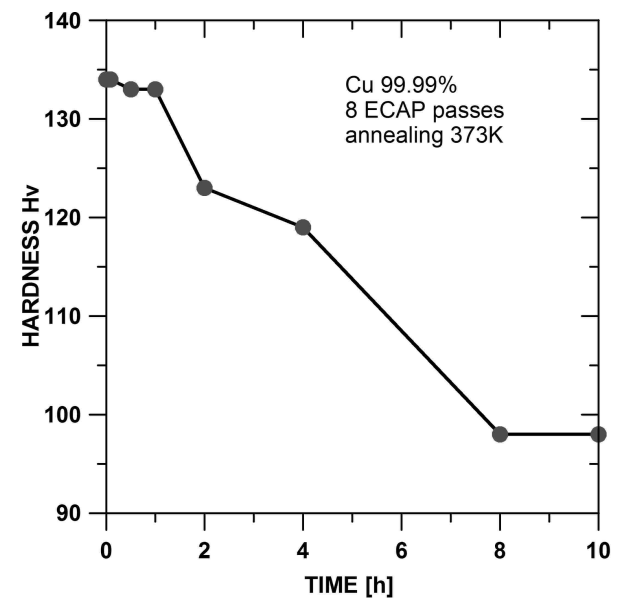

Fig. 2. Hardness of $\mathrm{Cu}$ processed by 8 ECAP passes and annealed at $373 \mathrm{~K}$ up to $10 \mathrm{~h}$.

The specimens for microstructure analyses were cut using electro spark process in an oil bath which minimalizes the effect of deformation and temperature on the surface. The specimens were grinded by 600 $4000 \mathrm{SiC}$ paper and water was used as the lubricant during grinding. The specimens were grinded by $600 \mathrm{SiC}$ paper until their surfaces were flat. The surfaces of grinded specimens were checked by light microscope Neophot 32. The specimens were rotated by $90^{\circ}$ between subsequent grinding steps and grinded perpendicular to the scratches which were created in the former grinding step until these scratches disappeared. Last grinding step was performed using $4000 \mathrm{SiC}$ paper in order to reduce time of electrolytic polishing. It is generally accepted that electropolishing is a widely used method for final step preparation because it removes the strains induced by mechanical grinding. Finally, the specimens were electropolished using $250 \mathrm{ml}$ phosphoric acid, $250 \mathrm{ml}$ ethanol, $50 \mathrm{ml}$ propylalcohol and $500 \mathrm{ml}$ water for $60 \mathrm{~s}$ at room temperature.

All the three cross sections $\mathrm{XY}, \mathrm{XZ}$ and $\mathrm{YZ}$ were examined but the analyses were especially focused on the section XZ. The area from which the EBSD patterns is acquired with an electron beam focused on a $70^{\circ}$ tilted sample, is approximately elliptical (Humphreys, 2001). For this reason, resolution perpendicular and parallel to the tilt axis can be distinguished, see Fig. 3 in Humphreys (2001). The major axis, which is perpendicular to the tilt axis, is about three times longer than the minor axis.

The electron beam is deflected and the orientation data are acquired and stored in each point of selected area. The point-to-point step size is based on the expected microstructure and examined size region. In the present work, the size of the image window was selected as $128 \times 96 \mu \mathrm{m}$ and the step of the EBSD was $\sim 0.5 \mu \mathrm{m}$ for the annealed specimens and these parameters were $40 \times 30 \mu \mathrm{m}$ and $\sim 0.07 \mu \mathrm{m}$ for the specimen only processed by ECAP.

Each EBSD pattern is analyzed and the solution is found when at least 4 diffraction lines are used for its determination. When the number of determined lines is lower the solution is not found and non-indexed data point is assigned. In the case that the number of nonindexing points is low, the data can be repaired by clean-up procedure. This procedure ensures that data points with the probability $>0.95$ of correct indexing are retained in the analysis. The points where the probability of correct indexing is lower (points with low pattern quality) are re-assigned to neighbouring regions of similar orientation. The procedure assumes that low pattern quality points are associated with grain boundaries or regions of high dislocation density. It is known that EBSD lines are not always ideal and for this reason, the standard angle difference of $2.5^{\circ}$ between acquired lines and lines of the solution is adjusted.

The quality map of EBSD patterns with the demonstration of an EBSD pattern is in Fig. 3. The light points denote high quality and the black points denote non-indexable EBSD patterns. The inspection of Fig. 3 shows that quality of EBSD patterns was high. The number of indexable EBSD patterns was approximately $97-98 \%$ in the annealed specimens and $79 \%$ in the specimens only processed by ECAP.

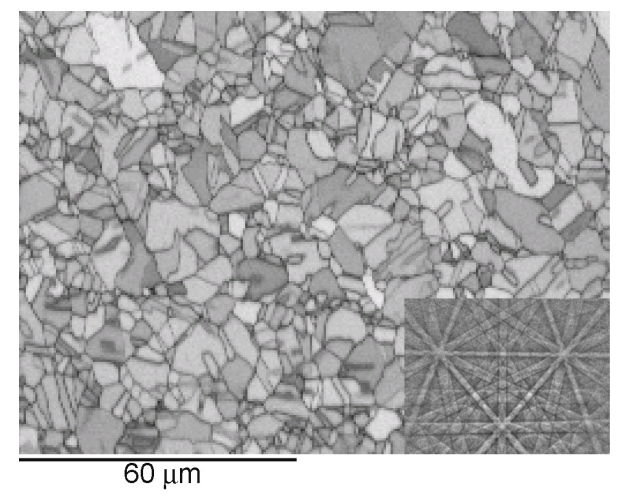

Fig. 3. EBSD pattern quality map of $C u$ annealed at $573 \mathrm{~K}$ for $10 \mathrm{~h}$ with the corresponding EBSD pattern. Window size $128 \times 96 \mu \mathrm{m}$, grid step $0.5 \mu \mathrm{m}$.

\section{IMAGE PROCESSING}

The main information measured by EBSD are Euler angles $\varphi_{1}, \Phi, \varphi_{2}$ representing the crystal orientation in each grid point (Fig. 4). These three parameters are sufficient to describe the mutual position of a reference coordinate system and orientation of the crystal lattice. 

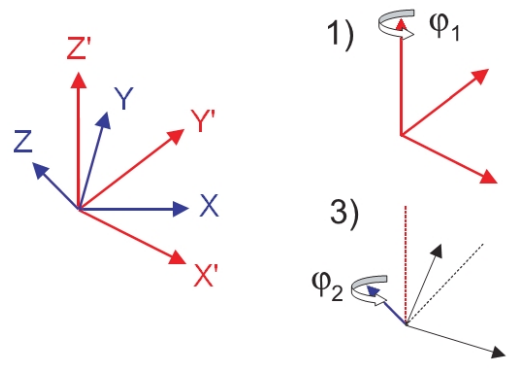

2)

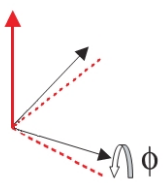

4)

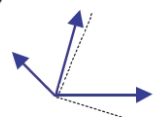

Fig. 4. Definition of the Euler angles $\varphi_{1}, \Phi, \varphi_{2}$. The crystal coordinate system (red) can be superimposed onto the sample system (blue) by three rotations: 1) rotation by $\varphi_{1}$ around the $Z$ axis of the crystal coordinate system, 2) rotation by $\Phi$ around the new $X$ axis and 3) rotation by $\varphi_{2}$ around the new $Z$ axis. The dotted lines show the positions of the axes before the last rotation.

Fig. 5a describes the definition of a crystal direction $[u v w]$. Further, it is common to denote $\langle u v w\rangle$ all crystalographically-related directions, i.e., the directions coincident with $[u v w]$ with respect to all symmetries of the crystal lattice. Supposing the difference in orientations between two neighbouring points which is called their misorientation, it is common to use an angle-axis representation $\theta\langle u v w\rangle$ based on the fact that one orientation can be matched to another using rotation by an angle $\theta$ around an axis $\langle u v w\rangle$. Because of non-uniqueness of this transformation especially in highly symmetric systems, only the solution with the minimum angle $\theta$ is considered and such a transformation $\theta\langle u v w\rangle$ is called disorientation. Results presented in this paper come from a research of metals with cubic crystal systems where the upper limit for the angle $\theta$ is about $62.8^{\circ}$. Details of conversion among Euler angles, transformation matrices and angle-axis representation can be found in (Engler and Randle, 2010).

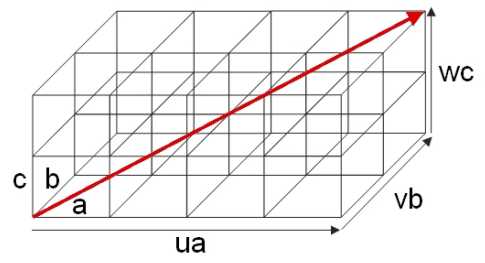

(a)

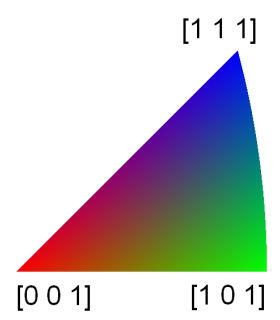

(b)
Fig. 5. (a) Meaning of a crystal direction $[u v w]$ in a crystal lattice with length parameters $a, b, c$ of a unit cell. The direction shown here is [422]. (b) Inverse pole figure as a colour scheme for orientation imaging.
Data obtained from EBSD can be immediately displayed in a pixel image where each pixel corresponds to one grid point and its colour is related to the crystal orientation (Fig. 9 in the section Numerical results). To represent each orientation by a single colour, an inverse pole figure shown in Fig. 5b is used as a colour scheme. Every orientation is located there according to the direction of one chosen axis of a reference coordinate system with respect to the crystal coordinate system in the given point. For instance, pure blue (vertex [111]) reveals that the chosen reference axis is parallel to the body diagonal of a unit cubic crystal cell. Because of invariance under rotations around the reference axis, this representation is not sufficient but still very illustrative for recognizing differences in orientations. Grain boundaries with the disorientation angle exceeding a limit value $\Delta$, which is equal to $15^{\circ}$ in Fig. 9, are coloured white. Another possibility often used is colouring the grains with random colours in order to distinguish them easily (Fig. 7).

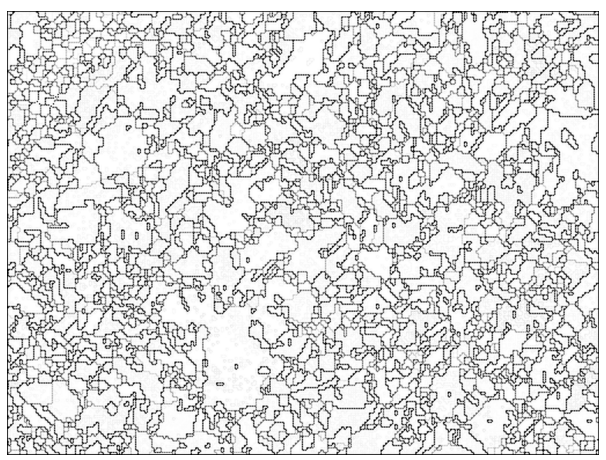

(a)

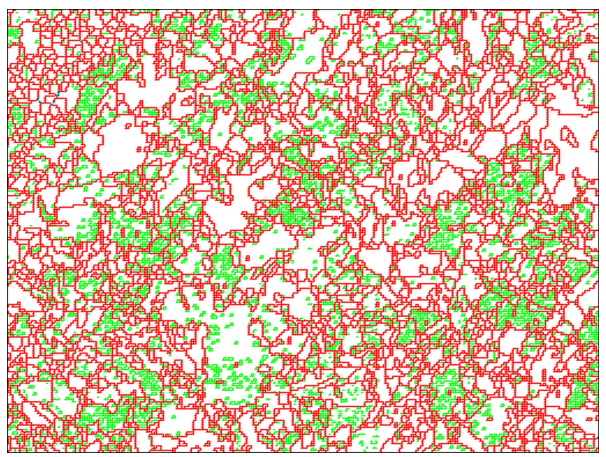

(b)

Fig. 6. Grain and subgrain boundaries in a sample of copper after 8 ECAP passes annealed for 10 hours at $373 \mathrm{~K}$, section $X Z$, window size $128 \times 96 \mu \mathrm{m}$, grid step $0.5 \mu \mathrm{m}$. (a) Darkness of a boundary corresponds to its disorientation angle $\theta$. Pure white for $\theta \leq$ $5^{\circ}$ changes linearly to pure black for $\theta=62.8^{\circ}$. (b) Boundaries are divided into two types - green low angle boundaries $\left(\theta \leq 15^{\circ}\right)$ and red high angle boundaries $\left(\theta>15^{\circ}\right)$. 
When focusing just on the grain boundaries, we can draw them dependently on the disorientation angle $\theta$. In Fig. 6a the darkness of each boundary is related to the disorientation angle; pure black colour corresponds to the maximum angle $62.8^{\circ}$. In Fig. $6 \mathrm{~b}$ two types of boundaries are distinguished - green low angle boundaries with $\theta \leq 15^{\circ}$ and red high angle boundaries with $\theta>15^{\circ}$. In a similar way it is also possible to visualize some special boundaries according to their coincidence site lattice (CSL) type.

\section{QUANTITATIVE ANALYSIS OF GRAIN BOUNDARIES}

To specify a grain boundary, five parameters are needed in general. Three of them are related to its misorientation (e.g., Euler angles) and the other two describe the orientation of the interface plane (e.g., spherical angles of the plane normal). However, characterizing the whole five-parameter distribution requires a large population of observable grain boundaries. In that case it is possible to use statistical methods developed for estimating the distribution density. For better insight, only changes in several parameters fixing the other ones are actually investigated which usually leads to some discretization of the parametrical space. In what follows, we will focus just on the misorientations of grain boundaries, especially the disorientation angle. This quantity represents a real-valued mark of the grain boundary network which allows to characterize it by the means of random marked closed sets (RMCS). In the following, the terminology and notation from (Ballani et al., 2012) are used.

We consider a random marked closed set $(Y, Z)$ in the $d$-dimensional Euclidean space $\mathbb{R}^{d}$ as a random function $Z$ defined on a random domain $Y \subset \mathbb{R}^{d}$. RMCS is stationary if its distribution is invariant under translations and it is isotropic if its distribution is invariant under rotations.

For any $\varepsilon \geq 0$ define the random field

$$
Z_{\mathcal{\varepsilon}}(x)=\left\{\begin{array}{cl}
\max _{y \in Y \cap B_{\varepsilon}(x)} Z(y) & \text { for } x \in Y_{\oplus \mathcal{\varepsilon}}, \\
0 & \text { otherwise }
\end{array}\right.
$$

where $B_{\varepsilon}(x)$ denotes the Euclidean ball in $\mathbb{R}^{d}$ with centre $x$ and radius $\varepsilon, Y_{\oplus \varepsilon}$ is a dilated set $Y_{\oplus \varepsilon}=Y \oplus$ $B_{\varepsilon}(o)$.

Second-order characteristics of RMCS $(Y, Z)$ for $x, y \in \mathbb{R}^{d}$ are defined as follows. Let us define

$$
\kappa_{f}(x, y)=\lim _{\varepsilon \downarrow 0} \mathbb{E}\left[f\left(Z_{\varepsilon}(x), Z_{\varepsilon}(y)\right) \mid x, y \in Y_{\oplus \varepsilon}\right],
$$

whenever $\kappa_{|f|}(x, y)<\infty$ and $\mathbb{P}\left(x, y \in Y_{\oplus \varepsilon}\right)>0$ for all $\varepsilon>0$, otherwise $\kappa_{f}(x, y)$ is undefined. Eq. 1 is a limit of conditional expectation given the two points $x, y$ lie in the dilated set. Common choices of $f$ are

$$
\begin{array}{ll}
e(m, n)=m, & c(m, n)=m n, \\
v(m, n)=m^{2}, & g(m, n)=(m-n)^{2} .
\end{array}
$$

Then define the conditional mean mark and the mark covariance function

$$
\begin{aligned}
E_{\kappa}(x, y) & =\kappa_{e}(x, y) \\
\operatorname{cov}_{\kappa}(x, y) & =\kappa_{c}(x, y)-\kappa_{e}(x, y) \kappa_{e}(y, x) .
\end{aligned}
$$

Further express the mark correlation function

$$
\operatorname{cor}_{\kappa}(x, y)=\frac{\kappa_{c}(x, y)-\kappa_{e}(x, y) \kappa_{e}(y, x)}{\sqrt{\left(\kappa_{v}(x, y)-\kappa_{e}(x, y)^{2}\right)\left(\kappa_{v}(y, x)-\kappa_{e}(y, x)^{2}\right)}}
$$

and the mark variogram

$$
\gamma_{\kappa}(x, y)=\frac{\kappa_{g}(x, y)}{2}=\frac{\kappa_{v}(x, y)+\kappa_{v}(y, x)}{2}-\kappa_{c}(x, y) .
$$

Another characteristic is the Stoyan's $k_{m m}$ function (Stoyan et al., 1995)

$$
k_{m m}(x, y)=\bar{m}^{-2} \kappa_{c}(x, y), \quad \bar{m}=\mathbb{E}[Z(x) \mid x \in Y] .
$$

Under the assumptions of stationarity and isotropy of the RMCS $(Y, Z)$, the characteristics defined in Eq. 1 are functions of the distance $r=\|x-y\|$ only. For their estimation on a bounded window $W \subset \mathbb{R}^{d}$, choose a finite set of test points $T \subset W$ such that for a fixed $\varepsilon>0$ and for suitable interpoint distances $r \in \mathbb{R}^{+}$, the sets

$$
\begin{aligned}
& N_{\mathcal{\varepsilon}, T}^{(1)}(r)=\left\{x \in Y_{\oplus \mathcal{E}} \cap T: \exists y \in Y_{\oplus \mathcal{E}} \cap T:\|x-y\|=r\right\} \\
& N_{\mathcal{\varepsilon}, T}^{(2)}(r)=\left\{(x, y) \in\left(Y_{\oplus \varepsilon} \cap T\right)^{2}:\|x-y\|=r\right\}
\end{aligned}
$$

are nonempty. For $\kappa_{e}, \kappa_{v}$ and $\kappa_{c}$ we use the following statistical estimators:

$$
\begin{aligned}
\widehat{\kappa}_{e}(r) & =\frac{1}{\left|N_{\varepsilon, T}^{(1)}(r)\right|} \sum_{x \in N_{\varepsilon, T}^{(1)}(r)} Z(x) \\
\widehat{\kappa}_{v}(r) & =\frac{1}{\left|N_{\varepsilon, T}^{(1)}(r)\right|} \sum_{x \in N_{\varepsilon, T}^{(1)}(r)} Z(x)^{2} \\
\widehat{\kappa}_{c}(r) & =\frac{1}{\left|N_{\varepsilon, T}^{(2)}(r)\right|} \sum_{(x, y) \in N_{\varepsilon, T}^{(2)}(r)} Z(x) Z(y) \\
\widehat{\kappa}_{g}(r) & =\frac{1}{\left|N_{\varepsilon, T}^{(2)}(r)\right|} \sum_{(x, y) \in N_{\varepsilon, T}^{(2)}(r)}(Z(x)-Z(y))^{2}
\end{aligned}
$$


where $|\cdot|$ stands for cardinality of the set. Under some additional assumptions, these estimators are asymptotically unbiased when $\varepsilon \downarrow 0$.

Taking into account the grain boundary structure with almost constant marks along its edges, it is reasonable to consider whether a given pair of points lies on the same edge or not. An important feature of the estimators introduced above is that marks in a small distance are highly correlated simply because of the fact that the pair of points often belongs to the same edge. However, correlations just among different edges can give us more valuable information about the second-order structure. In what follows, we denote by $x \sim y$ the relation that the points $x$ and $y$ belong to $\varepsilon$ neighbourhood of one edge and by $x \neq y$ the opposite case. Under stationarity and isotropy we define

$$
\begin{aligned}
& \tilde{N}_{\varepsilon, T}^{(1)}(r)=\left\{x \in N_{\mathcal{\varepsilon}, T}^{(1)}(r): \exists y \in N_{\varepsilon, T}^{(1)}(r): x \nsim y\right\} \\
& \tilde{N}_{\varepsilon, T}^{(2)}(r)=\left\{(x, y) \in N_{\varepsilon, T}^{(2)}(r): x \nsim y\right\}
\end{aligned}
$$

and $\widehat{\widetilde{\kappa}}_{e}(r), \widehat{\widetilde{\kappa}}_{v}(r), \widehat{\widetilde{\kappa}}_{c}(r), \widehat{\widetilde{\kappa}}_{g}(r)$ can be defined in a similar way like Eqs. 6-9, using non edge-related sets $\tilde{N}_{\varepsilon, T}^{(1)}(r), \tilde{N}_{\varepsilon, T}^{(2)}(r)$ instead of $N_{\varepsilon, T}^{(1)}(r), N_{\varepsilon, T}^{(2)}(r)$. Benefit of these estimators is that they suppress the effect of high correlation of pairs of points belonging to the same edge.

Another approach to characterizing the grain boundary structure as a marked fibre process consists in investigating several types of special boundaries dominantly influencing properties of the material. Let us suppose that each point of the grain boundary network is given a categorical mark $Z(x) \in \mathscr{Z}=$ $\{1,2, \ldots, m\}$ which is constant for points belonging to the same edge. For each pair of values $i, j \in \mathscr{Z}$, we define the cross K-function

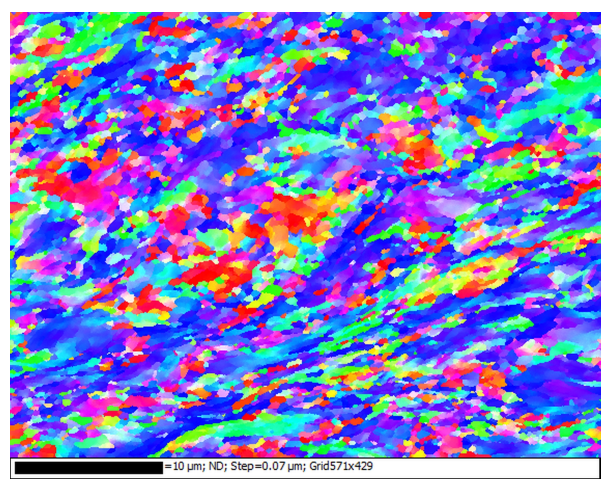

Fig. 7. Crystal orientation map of $\mathrm{Cu}$ after 8 ECAP passes with randomly coloured grains. Window size $40 \times 30 \mu \mathrm{m}$, grid step $0.07 \mu \mathrm{m}$.

$$
\begin{array}{r}
K_{i, j}(r)=\frac{1}{\lambda_{i} \lambda_{j} \delta(A)} \mathbb{E}\left[\int_{Y} \int_{Y \cap A} 1_{[\|x-y\| \leq r]} \mathrm{d} x \mathrm{~d} y \mid\right. \\
Z(x)=i, Z(y)=j],
\end{array}
$$

where $\lambda_{i}$ is an intensity function of a fibre subprocess of edges with the mark $i \in \mathscr{Z}$ and $A \in \mathscr{B}_{0}$ is an arbitrary bounded Borel set with positive Lebesgue measure $\delta(A)>0$. To estimate Eq. 10 it is necessary to provide a segmentation of pixellated grain boundaries (Arnould et al., 2001; Jeulin and Moreaud, 2008). During this smoothing procedure, the grain boundaries are identified as lines or curves separating different phases in the image. On the segmented fibre structure $Y_{s}$ we firstly define a simple intensity estimator of $\lambda_{i}$

$$
\widehat{\lambda}_{i}=\frac{1}{\delta(W)} \int_{Y_{S}} 1_{[Z(y)=i]} \mathrm{d} y .
$$

Based on the set of test points $V_{i} \subset\left\{y \in Y_{s}: Z(y)=i\right\}$, an estimator of Eq. 10 can be expressed as

$$
\widehat{K_{i, j}}(r)=\frac{\sum_{x \in V_{i, \ominus r}}\left(\int_{Y_{s}} 1_{[\| x-y|| \leq r, Z(y)=j]} \mathrm{d} y\right)}{\hat{\lambda}_{i} \hat{\lambda_{j}} \delta\left(W_{\ominus r}\right)\left|V_{i, \ominus r}\right|},
$$

where $W_{\ominus r}=W \ominus B_{r}(o)$ is eroded window providing the edge effects correction and $V_{i, \ominus r}=V_{i} \cap W_{\ominus r}$.

The K-function can be useful for interpretation of clustering of different types of grain boundaries. For instance, higher values of $K_{i, j}(r)$ indicate clustering of edges with the mark $j$ around edges with the mark $i$.

\section{NUMERICAL RESULTS}

The methods were applied to data obtained from EBSD of copper processed by equal-channel angular

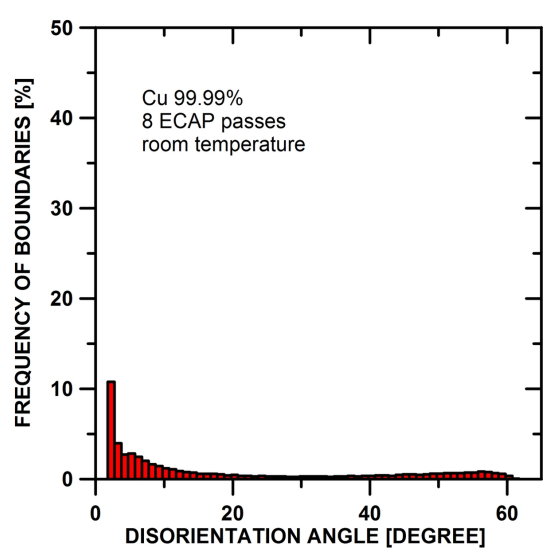

Fig. 8. Histogram of disorientation angles of $\mathrm{Cu}$ after 8 ECAP passes. 

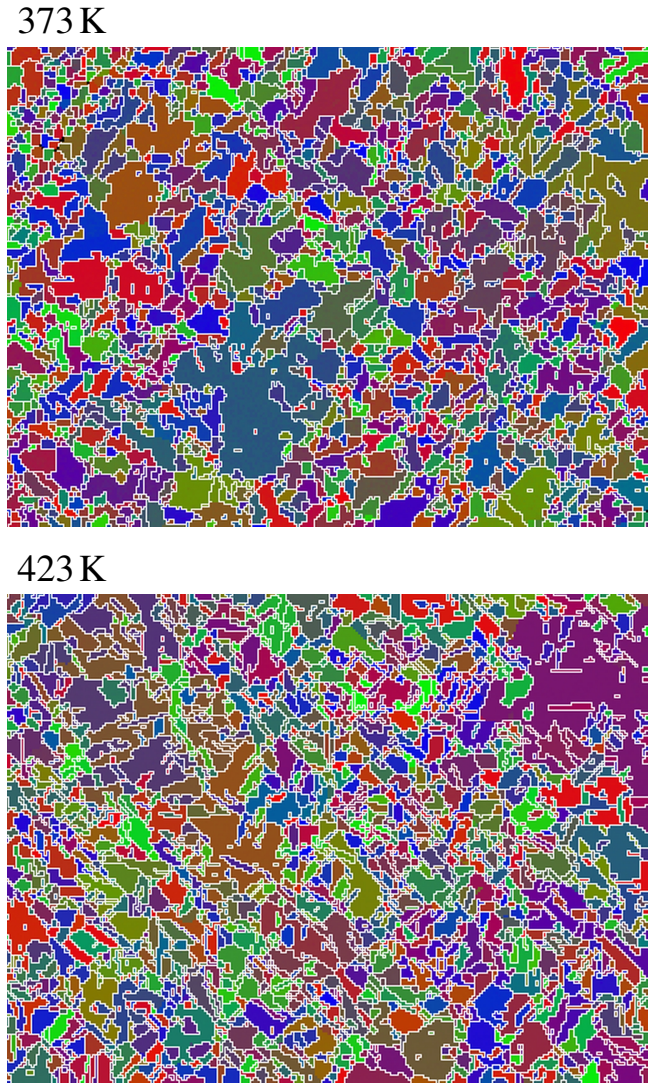

$473 \mathrm{~K}$

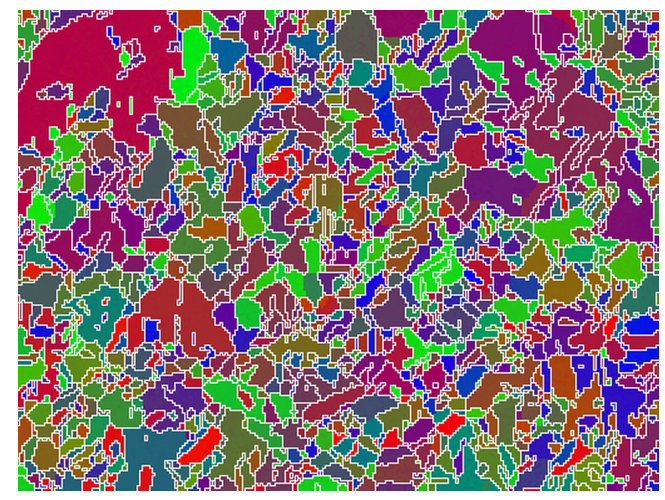

$573 \mathrm{~K}$

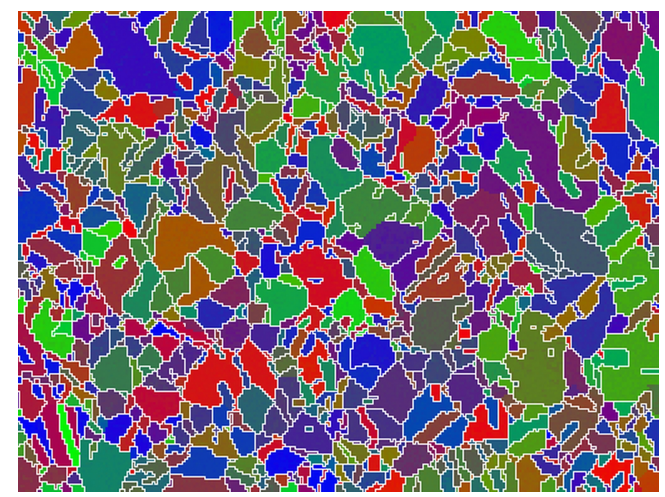

Fig. 9. Crystal orientation maps of $\mathrm{Cu}$ after 8 ECAP passes annealed for 10 hours at $373 \mathrm{~K}, 423 \mathrm{~K}, 473 \mathrm{~K}$ or $573 \mathrm{~K}$. Window size $128 \times 96 \mu \mathrm{m}$, grid step $0.5 \mu \mathrm{m}$.
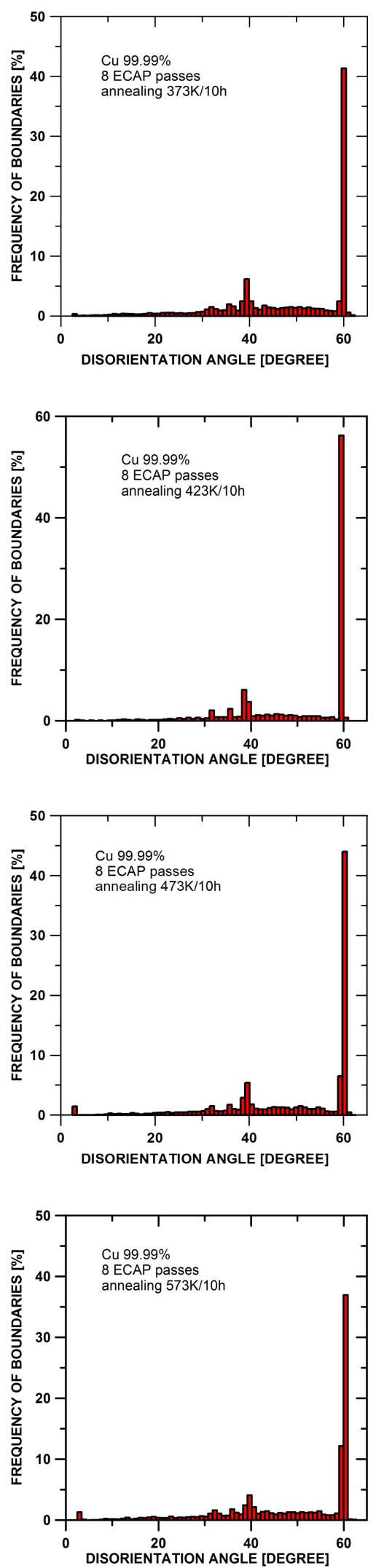

Fig. 10. Histograms of disorientation angles of four examined samples from Fig. 9. 
pressing. After 8 passes through the die, the samples were annealed for 10 hours at different temperatures - $373 \mathrm{~K}, 423 \mathrm{~K}, 473 \mathrm{~K}$ and $573 \mathrm{~K}$ (Fig. 9). It is obvious that the temperature of annealing influences the microstructure.

To indicate changes in the average grain size, Table 1 summarizes the mean areas of grain profiles and their variation coefficients, i.e., ratio of the standard deviation and the mean area, in observed cross section.

Table 1. Mean profile areas in $\mu m^{2}$ and their variation coefficients in the sample without annealing and four annealed samples.

\begin{tabular}{crrrr}
\hline no annealing & $373 \mathrm{~K}$ & $423 \mathrm{~K}$ & $473 \mathrm{~K}$ & $573 \mathrm{~K}$ \\
\hline 0.97 & 4.90 & 3.21 & 7.01 & 10.57 \\
0.01 & 0.20 & 0.26 & 0.29 & 0.12 \\
\hline
\end{tabular}

Increasing tendency in average grain size also influences the lengths of the edges of the grain boundaries observed in the cross sections. Fig. 11 shows that the majority of them lies below $2 \mu \mathrm{m}$ (edges shorter than $0.5 \mu \mathrm{m}$ were excluded during the segmentation) but especially in the last sample even several times longer edges are present.

Histograms of disorientation angles in Fig. 10 indicate among others a high fraction of $\Sigma 3$ boundaries with $\theta=60^{\circ}$, mostly being so called twin boundaries with axis of rotation $\langle 1,1,1\rangle$, and $\Sigma 9$ boundaries with $\theta \doteq 39^{\circ}$. However, in the microstructure of copper processed by 8 ECAP passes rather low-angle grain boundaries (LAGB's) and random high-angle grain boundaries (HAGB's) predominated. From Table 2 it is apparent that frequency of random HAGB's remains about $40-50 \%$ while frequency of twin boundaries rapidly increases when the material is annealed.

Table 2. Frequency of selected boundaries in microstructure of pure copper processed by ECAP and subsequent annealing at different temperatures for $10 h$.

\begin{tabular}{crrrr}
\hline specimen & LAGB's & $\Sigma 3$ & $\Sigma 9$ & HAGB's \\
\hline no annealing & 57.92 & 2.57 & 0.25 & 39.26 \\
$373 \mathrm{~K}$ & 2.50 & 35.83 & 6.75 & 54.92 \\
$423 \mathrm{~K}$ & 2.10 & 54.30 & 7.42 & 36.18 \\
$473 \mathrm{~K}$ & 2.89 & 41.43 & 6.15 & 49.53 \\
$573 \mathrm{~K}$ & 3.08 & 40.13 & 5.09 & 51.70 \\
\hline
\end{tabular}

Observation of high frequency of twins is fully consistent with the investigation of the role of shear stress in formation of annealing twin boundaries in copper (Field et al., 2006). Field et al. revealed that the twin content in rolled copper with $92 \%$ reduction is significantly lower than that in any copper deformed by ECAP, regardless of the annealing temperature. (Molodova et al., 2007) found a very low thermal stability of pure copper processed by ECAP. They observed that in the microstructure of pure copper processed by 12 ECAP passes, large recrystallized grains can be already found even after annealing at $393 \mathrm{~K}$ for $10 \mathrm{~min}$ and $423 \mathrm{~K}$ for $2 \mathrm{~min}$. In our study, the occurrence of large recrystallized grains was observed at all annealed temperatures with markedly local character.

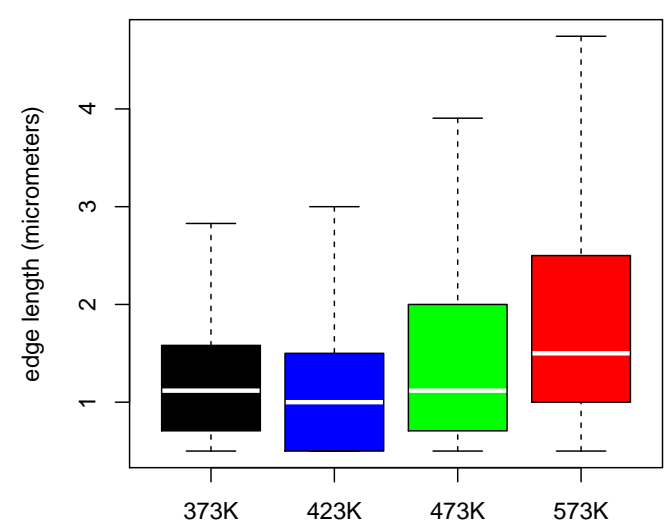

Fig. 11. Box plots of lengths of the grain boundaries of four examined samples. The plots show median, the first and the third quartile and 1.5 multiple of the interquartile range. Outliers are not figured.

Occurrence of high frequency of twin boundaries in the microstructure of annealed copper samples before loading in the creep testing machine can significantly influence creep behaviour. It was found (Watanabe and Tsurekawa, 1999; Watanabe, 2011) that a high fraction of strong low- $\Sigma$ boundaries is a key factor controlling intergranular brittleness. The control of intergranular fracture and intergranular brittleness can be achieved by reduction of random boundaries or conversely by increasing the fraction of LAGB's or special low $\Sigma$ coincidence boundaries resistant to fracture.

It is generally accepted that the damage near grain boundaries is one of the key factors controlling creep life because many cracks are initiated at grain boundaries and frequently major degradation phenomena in materials are subjected to the creep exposure. Furthermore, grain boundaries can influence creep behaviour of ultrafine-grained materials due to synergetic effect of additional operating creep mechanisms like grain boundary sliding (GBS), intergranular cavitation or more intensive grain 
boundary diffusion (Král et al., 2012). Nevertheless the ability of GBS takes place more significantly at a random grain boundary compared with low- $\Sigma$ boundaries (Kokawa et al., 1981; Watanabe et al., 1984).

With the characteristics introduced in the previous section, we aim at characterizing the microstructure in a more complex view. The following results show that even though the marginal distribution of disorientation angles and the fraction of special boundaries can be similar, their arrangement in the grain boundary network can differ crucially. The second-order characteristics describe these aspects on the basis of correlations and clustering of different grain boundary types measured in a small distance radius. The quantities defined in Eq. 1 are estimated using a set of test points $T$ given by all the grid points of the EBSD measurement and a fixed $\varepsilon>0$ given by the grid step. The cross K-function Eq. 10 is estimated using a set of test points $V_{i}$ given by midpoints of the $i$-th type boundaries, $i \in \mathscr{Z}=\{3,9, L, H\}$, where the marks correspond to $\Sigma 3, \Sigma 9$, LAGB's and random HAGB's.
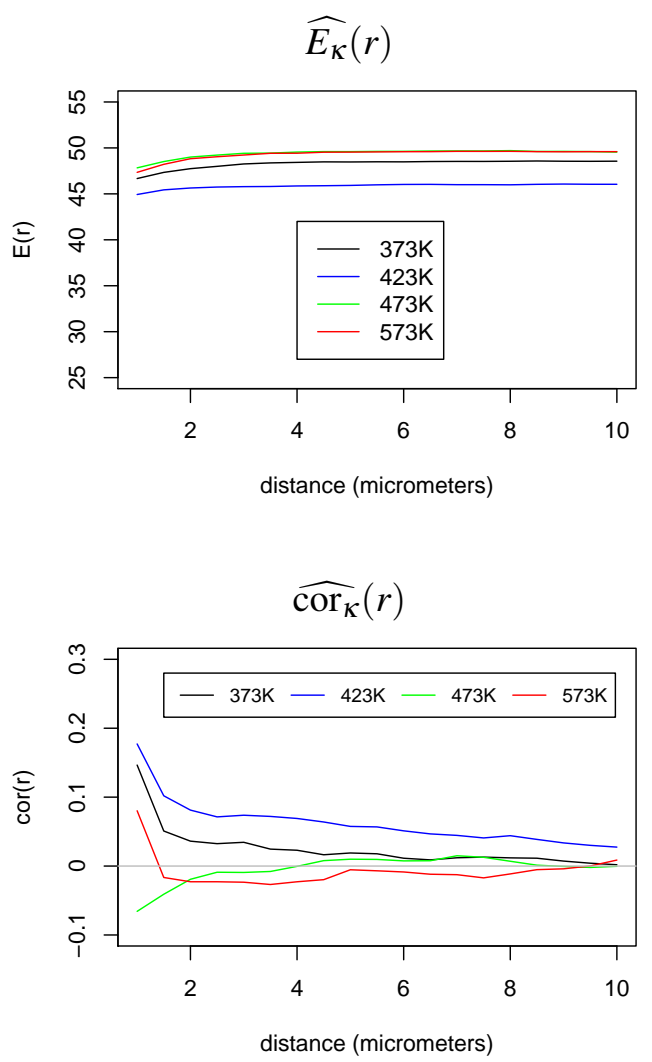

Fig. 12. Estimators of the mark expectation $\widehat{E_{\kappa}}(r)=$ $\widehat{\widetilde{\kappa}}_{e}(r)$ and the mark correlation function $\widehat{\operatorname{cor}_{K}}(r)=$ $\widehat{\widetilde{\kappa}}_{c}(r)-\widehat{\widetilde{\kappa}}_{e}(r)^{2}$ offour examined samples.
Fig. 12 shows the estimators of the mark expectation $\widehat{E_{K}}(r)=\widehat{\widetilde{\kappa}}_{e}(r)$ and the mark correlation function $\widehat{\operatorname{cor}}_{\kappa}(r)=\widehat{\widetilde{\kappa}}_{c}(r)-\widehat{\widetilde{\kappa}}_{e}(r)^{2}$ of four examined samples. The first estimator is almost constant because for every step distance $r$ plotted there, the set $\tilde{N}_{\varepsilon, T}^{(1)}(r)$ contains almost every point $x \in Y_{\oplus \varepsilon} \cap T$ and in this case the estimator equals to the unconditional mean mark with respect to $T$. With increasing annealing temperature, we can observe increasing trend of the mark expectation and decreasing trend of the mark correlation function. In other words, increase in the annealing temperature leads to more random arrangements of edges within the distance $r$ in the sense of their disorientation angles.

In the next we aim at localization of the dominant $\Sigma 3$ boundaries near the other boundary types. Fig. 13 shows the estimators of the cross K-functions $\widehat{K_{3,3}}(r)$, $\widehat{K_{9,3}}(r), \widehat{K_{L, 3}}(r)$ and $\widehat{K_{H, 3}}(r)$, which help to interpret the occurrence of $\Sigma 3$ boundaries in neighbourhoods of $\Sigma 3, \Sigma 9$, LAGB's or random HAGB's. While Table 2 shows the marginal proportions of different boundary types, these K-functions bring an additional information about their mutual positions in the structure. We see that in the samples with higher annealing temperature, values $\widehat{K_{3,3}}(r)$ and $\widehat{K_{9,3}}(r)$ are generally lower which indicates higher regularity of $\Sigma 3$ boundaries with respect to themselves or $\Sigma 9$ boundaries. On the other hand, the situation is different in the neighbourhood of less dominant boundary types where these functions are minimal for the lowtemperature annealed $423 \mathrm{~K}$ sample but any clearly interpretable trend is missing here.

It was found (Molodova et al., 2007; Saxl et al., 2010) that application of ECAP method could lead to the formation of the bimodal or even multimodal microstructures. The bimodality can depend on an appropriate thermal or creep loading conditions. It is widely accepted that the co-existence of larger recrystallized grains in the bimodal structure can improve deformation behaviour and thereby a ductility of ultrafine-grained material by relaxation of the stress concentration, created by GBS, through plastic deformation inside of larger grains (Ma, 2003; Koch, 2003; Fan et al., 2006). By contrast, a very recent report on creep ductility of ultrafine-grained materials did not confirm general acceptance of this view (Sklenička et al., 2012).

\section{CONCLUSIONS}

The present paper defines the grain boundary structure as a random marked closed set which 

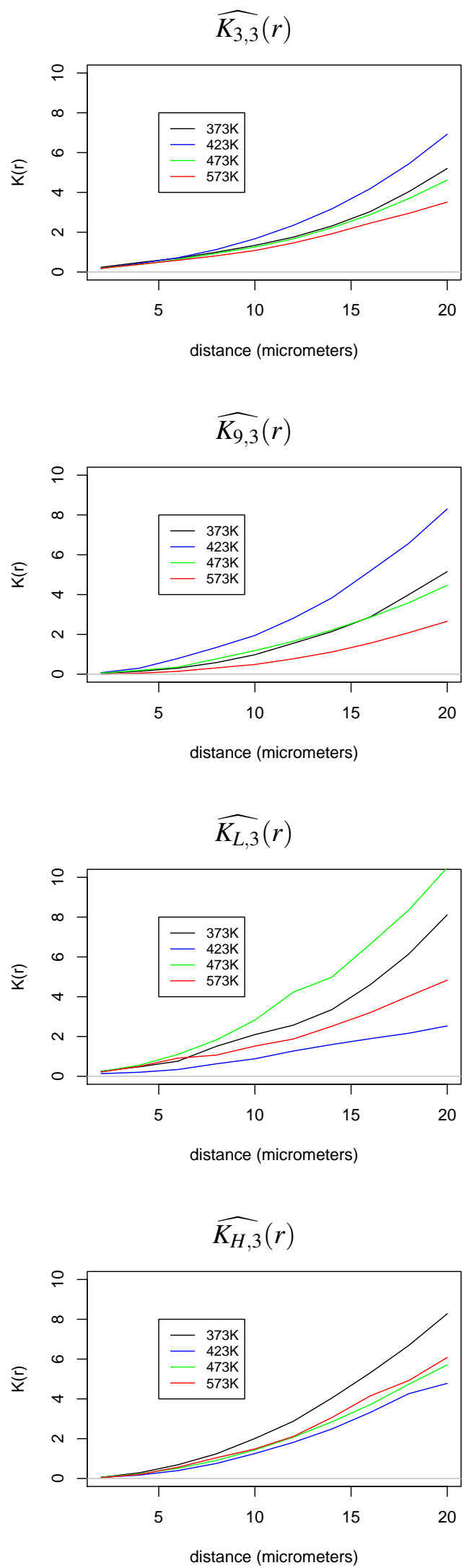

Fig. 13. Estimators of the cross $K$-functions $\widehat{K_{3,3}}(r)$, $\widehat{K_{9,3}}(r), \widehat{K_{L, 3}}(r)$ and $\widehat{K_{H, 3}}(r)$ of four examined samples where the indexes 3,9,L,H represent $\Sigma 3, \Sigma 9, L A G B$ 's and random $H A G B$ 's. is observable in a planar section with the use of orientation imaging microscopy. To characterize its spatial distribution, it is useful to extend the common attitude based on marginal distributions to the secondorder analysis. Methods of estimation of the secondorder characteristics are provided and their use on particular specimens of metallic material is shown. To reveal the dependency between disorientations as a function of distance in a stationary and isotropic structure, appropriate estimators of secondorder characteristics of the marks are defined which suppress the effect of high correlation of the marks along particular edges.

The methods are demonstrated on grain boundary structures marked by the disorientation angle or equipped with categorical marks indicating specialness of boundaries according to their CSL type. The subsequent annealing of the microstructure of pure copper processed by 8 ECAP passes led to the formation of the bimodal microstructure containing high fraction of low- $\Sigma$ coincidence boundaries. The second-order characteristics provide an additional information about arrangements of different boundary types in the structure. Our results show that increasing temperature of annealing leads to decreasing tendency of $\Sigma 3$ boundaries to form clusters but more likely to be placed regularly or create longer paths in the microstructure.

\section{ACKNOWLEDGEMENT}

The research was supported by the Czech Science Foundation, project P201/10/0472, and Operational Program Research and Development for Innovations co-funded by the European Regional Development Fund (ERDF) and national budget of the Czech Republic, within the framework of project Centre of Polymer Systems (reg. number: CZ.1.05/2.1.00/03.0111). Special thanks belong to Jiří Dvořák for preparation of the samples and operation with the ECAP facilities. The topic of this paper was presented at the S4G Conference, June 25-28, 2012 in Prague, Czech Republic.

\section{REFERENCES}

Arnould X, Coster M, Chermant J-L, Chermant L, Chartier T, Elmoataz A (2001). Segmentation and grain size of ceramics. Image Anal Stereol 20:131-5.

Ballani F, Kabluchko Z, Schlather M (2012). Random marked sets. Adv Appl Probab 44(3):603-16.

Bastos A, Zaefferer S, Raabe D, Schuh C (2006). Characterization of the microstructure and texture of nanostructured electrodeposited NiCo using electron backscatter diffraction (EBSD). Acta Mater 54:245162. 
Britton TB, Wilkinson AJ (2012). Stress fields and geometrically necessary dislocation density distributions near the head of a blocked slip band. Acta Mater 60:5773-82.

Calcagnotto M, Ponge D, Demir E, Raabe D (2010). Orientation gradients and geometrically necessary dislocations in ultrafine grained dual-phase steels studied by $2 \mathrm{D}$ and 3D EBSD. Mater Sci Eng A 527:2738-46.

Dvořák J, Sklenička V, Král P, Svoboda M, Saxl I (2010). Characterization of creep behaviour and microstructure changes in pure copper processed by equal-channel angular pressing. Part I. Creep behaviour. Rev Adv Mater Sci 25:225-32.

Engler O, Randle V (2010). Introduction to texture analysis - macrotexture, microtexture and orientation mapping. 2nd Ed. CRC Press.

Fan GJ, Choo H, Liaw PK, Lavernia EJ (2006). Plastic deformation and fracture of ultrafine-grained $\mathrm{Al}-\mathrm{Mg}$ alloys with a bimodal grain size distribution. Acta Mater 54:1759-66.

Field DP, Eames RC, Lillo TM (2006). The role of shear stress in the formation of annealing twin boundaries in copper. Scripta Mater 54:983-6.

Furukawa M, Iwahashi I, Horita Z, Nemoto M, Langdon TG (1998). The shearing characteristics associated with equal-channel angular pressing. Mater Sci Eng A 257: 328-32. .

Humphreys FJ (2001). Grain and subgrain characterisation by electron backscatter diffraction. J Mater Sci 36:3833-54.

Ilucová L, Saxl I, Svoboda M, Sklenička V, Král P (2007). Structure of ECAP aluminium after different number of passes. Image Anal Stereol 26:37-43.

Jeulin D, Moreaud M (2008). Segmentation of 2D and 3D textures from estimates of the local orientation. Image Anal Stereol 27:183-92.

Jiang J, Godfrey A, Liu W, Liu Q (2008). Microtexture evolution via deformation twinning and slip during compression of magnesium alloy AZ31. Mater Sci Eng A 483-4:576-9.

Koch CC (2003). Optimization of strength and ductility in nanocrystalline and ultrafine grained metals. Scripta Mater 49:657-62.

Kokawa H, Watanabe T, Karashima S (1981). Sliding behaviour and dislocation structures in aluminium grain boundaries. Philos Mag A 44:1239-54.

Král P, Dvořák J, Kvapilová M, Svoboda M, Beneš V, Ponížil P, Šedivý O, Sklenička V (2011). Quantitative characterization of microstructure in copper processed by equal-channel angular pressing. Mat Sci Forum 6679:235-40.

Král P, Svoboda M, Dvořák J, Kvapilová M, Sklenička V (2012). Microstructure mechanisms governing the creep life of ultrafine-grained $\mathrm{Cu}-0.2 \mathrm{wt}$.\% $\mathrm{Zr}$ alloy. Acta Phys Pol A 122:457-60.

Ma E (2003). Instabilities and ductility of nanocrystalline and ultrafine-grained metals. Scripta Mater 49:663-8.

Molodova X, Gottstein G, Winning M, Hellmig RJ (2007). Thermal stability of ECAP processed pure copper. Mater Sci Eng A 460-1:204-13.

Perez M, Kenik E, Keefe OM, Miller F, Johnson B (2006). Identification of phases in zinc alloy powders using electron backscatter diffraction. Mater Sci Eng A 424:239-50.

Randle V, Rohrer G, Miller H, Coleman M, Owen G (2008). Five-parameter grain boundary distribution of commercially grain boundary engineered nickel and copper. Acta Mater 56:2363-73.

Randle V (2009). Electron backscatter diffraction: strategies for reliable data acquisition and processing. Mater Charact 60:913-22.

Rohrer GS (2011). Measuring and interpreting the structure of grain-boundary networks. J Am Ceram Soc 94(3):633-46.

Saxl I, Sklenička V, Ilucová L, Svoboda M, Král P, Dvořák J (2010). Characterization of creep behaviour and microstructure changes in pure copper processed by equal-channel angular pressing. Part II. The microstructural characteristics. Rev Adv Mater Sci 25: 233-40.

Sklenička V, Dvořák J, Král P, Svoboda M, Kvapilová M, Langdon TG (2012). Factors influencing creep flow and ductility in ultrafine-grained metals. Mat Sci Eng A 558:403-11.

Stoyan D, Kendall WS, Mecke J (1995). Stochastic geometry and its applications. 2nd Ed. New York: Wiley.

Watanabe T (2011). Grain boundary engineering: historical perspective and future prospects. J Mater Sci 46:4095115.

Watanabe T, Kimura S-I, Karashima S (1984). The effect of a grain boundary structural transformation on sliding in $\langle 1010\rangle$-tilt zinc bicrystals. Philos Mag A 49:845-64.

Watanabe T, Tsurekawa S (1999). The control of brittleness and development of desirable mechanical properties in polycrystalline systems by grain boundary engineering. Acta Mater 47:4171-85.

Wilkinson AJ, Britton TB (2012). Strains, planes, and EBSD in materials science. Mater Today 15:366-76. 